\title{
EXAMPLES OF REFLECTION POSITIVE EUCLIDEAN FIELD THEORIES
}

\author{
R. TRINCHERO
}

\begin{abstract}
The requirement of reflection positivity(RP) for Euclidean field theories is considered. This is done for the cases of a scalar field, a higher derivative scalar field theory and the scalar field theory defined on a non-integer dimensional space(NIDS). It is shown that regarding RP, the analytical structure of the corresponding Schwinger functions plays an important role. For the higher derivative scalar field theory RP does not hold. However for the scalar field theory on a NIDS, RP holds in a certain range of dimensions where the corresponding Minkowskian field is defined on a Hilbert space with a positive definite scalar product that provides a unitary representation of the Poincaré group. In addition, and motivated by the last example, it is shown that, under certain conditions, one can construct non-local reflection positive Euclidean field theories starting from the corrected two point functions of interacting local field theories.
\end{abstract}

\section{INTRODUCTION}

Given a Euclidean field theory defined by its $n$-point Schwinger functions, the OsterwalderSchröder (OS) axioms[1] allow to reconstruct a field theory on Minkowski space satisfying Wightman axioms. One of the OS axioms involves the condition of reflection positivity $(\mathrm{RP})$, this condition enables the definition of a positive semi-definite scalar product in Minkowski space. This, together with the other axioms, make possible to produce a set of Wightman functions which transform under unitary representations of the Poincaré group. In this work standard and non-standard examples of two point Schwinger functions are considered. These are the free scalar field, a higher derivative regulated scalar field and a scalar field in a NIDS. The example involving the higher derivative scalar field shows that the pole structure of the two point function plays a important role in determining the states of this system and their norms. The idea of extending the number of dimensions to non-integers appears in physics in two different fields, the Wilson renormalization group[2] and dimensional regularization[3][4]. From the mathematical point of view a general theory that can describe NIDS is given by non-commutative geometry 5 . The application of non-commutative geometry to NIDS's as required for the applications 11 mentioned above appears in[7]. In that reference a self-interacting scalar field is considered, the dimension $d$ of that NIDS is given by,

$$
d=\frac{n}{1-2 \alpha}
$$

where $n$ is the integer dimension of the space that is deformed and $\alpha \in \mathbb{R}$ the parameter controlling the deformation. In that reference it is shown that when $\alpha \neq 0$ the one-loop diagrams in these spaces are finite and the singularity structure for $\alpha \rightarrow 0$ is the same as in dimensional regularization. An important point, which was not addressed in that reference, is whether the theory in that space is unitary or not. In other words whether one

Date: $14 / 07 / 2017$.

${ }^{1}$ For its application to fractal systems see [5], [6] 
can define a physical theory in these spaces or that they only provide a regularization of the corresponding integer dimensional theories. As mentioned above reflection positivity is a key property in this respect and that is the reason for considering its validity. It turns out that RP holds in this case for $0<\alpha<\frac{1}{n+2}$. For $\alpha<0$ the space does not fulfill the conditions required by NCG, however one can consider the resulting field theory for these $\alpha$ values. It is shown that for $\alpha<0$ RP does not hold.

In addition, unlike the integer dimensional case the two point function in the NIDS in momentum space presents a branch cut in the complex $p^{2}$ plane. The appearance of this branch cut is similar to what happens in a interacting integer dimensional local field theory when considering loop corrections to the two point function. Motivated by this similarity the following question is addressed: Under what conditions the corrected Euclidean propagator coming from a local interacting field theory leads to a scalar product satisfying the requirement of reflection positivity?

The features and results of this work are summarized as follows,

- Examples of Euclidean field theories are considered and the condition of RP is checked for each case.

- For the higher derivative scalar field, which involves more than one pole for the two point function in momentum space, the results makes sense if each pole is considered separately.

- RP does not hold for the case of the scalar field in the NIDS if $\alpha<0$. However RP holds for $0<\alpha<\frac{1}{n+2}$. For this case the two point function in momentum space presents a branch cut, similar to what happens in a interacting local field theory.

- Motivated by the similarity mentioned in the previous item, it is shown that a corrected propagator in a local interacting unitary field theory leads a scalar product satisfying RP whenever the particle associated to that field can not decay.

The paper is organized as follows. Section 2 presents some general facts about RP and checks this condition for each of the above mentioned cases. Section 3 presents the scalar product and energy for each case. Section 4 studies the conditions under which a perturbatively defined interacting and unitary local field theory leads to a corrected propagator satisfying RP. Section 5 collects some concluding remarks.

\section{REFLECTION POSITIVITY}

In the framework of Euclidean functional integrals [8], reflection positivity can be phrased as follows,

$$
<(\Theta \mathcal{F}[\varphi]) \mathcal{F}[\varphi]>\geq 0
$$

where $\mathcal{F}[\varphi]$ denotes a functional of the fields with support in a region whose points have $x_{d+1}>0$ and $\Theta \mathcal{F}[\varphi]$ denotes the same functional complex conjugated and reflected respect to the $x_{d+1}=0$ plane. The brackets denote the expectation value respect to $S[\varphi]$, i.e.,

$$
<(\Theta \mathcal{F}[\varphi]) \mathcal{F}[\varphi]>=\frac{1}{\int \mathcal{D} \varphi e^{-S[\varphi]}} \int \mathcal{D} \varphi e^{-S[\varphi]}(\Theta \mathcal{F}[\varphi]) \mathcal{F}[\varphi]
$$

For a free theory or a perturbatively defined interacting theory, it suffices to consider the case when the functional $\mathcal{F}[\varphi]$ is linear 2 in $\varphi$ and $S[\varphi]$ is quadratic in the field and its

\footnotetext{
${ }^{2}$ Reflection positivity for higher order monomials follows using Wick's theorem.
} 
derivatives. This linear functional is written as,

$$
\mathcal{F}[\varphi]=\int d^{d+1} x f(x) \varphi(x)
$$

where $f(x)$ has support on points $x \in \mathbb{R}_{d+1}^{+}$, where $\mathbb{R}_{d+1}^{+}$denotes the points of $\mathbb{R}_{d+1}$ such that $x_{d+1}>0$. Defining the functional,

$$
G[J]=\int \mathcal{D} \varphi e^{-S_{J}[\varphi]}, S_{J}[\varphi]=S[\varphi]+\int d^{d+1} x J \varphi
$$

the expectation value appearing in the l.h.s. of (2.1) is,

$$
\begin{aligned}
<(\Theta \mathcal{F}[\varphi]) \mathcal{F}[\varphi]> & =\left.\frac{1}{G[0]} \int d^{d+1} x d^{d+1} y \bar{f}(\theta x) f(y) \frac{\delta}{\delta J(x)} \frac{\delta}{\delta J(y)} G[J]\right|_{J=0} \\
& =\int d^{d+1} x d^{d+1} y \bar{f}(\theta x) S(x-y) f(y) \\
& =\int d^{d+1} x d^{d+1} y \bar{f}(x) S(\theta x-y) f(y)
\end{aligned}
$$

where $S(x-y)$ denotes the Green function of the operator in the quadratic from appearing in $S[\varphi]$ and the bar indicates complex conjugation. Thus for this case, the requirement of reflection positivity is equivalent to,

$$
(\theta f, f) \geq 0, \text { Support }(f) \subset \mathbb{R}_{d+1}^{+}
$$

where the scalar product $($,$) is defined by,$

$$
(f, g)=\int d^{d+1} x d^{d+1} y \bar{f}(x) S(x-y) g(y)
$$

and $\theta f=f(\theta x), \theta x=\left(-x_{d+1}, \bar{x}\right)$. It is noted that this scalar product is defined not only for sources $f(x)$ with support on $\mathbb{R}_{d+1}^{+}$but also for sources with support in $\mathbb{R}_{d+1}$. The last two equations define what is sometimes referred to as the reflection positivity requirement for a one particle system[9] or the RP requirement for a bilinear form acting on a inner product space[10]. Motivated by the previous construction the external sources $f(x)$ are taken to belong to the Euclidean Hilbert space $\mathcal{E}$ of those tempered distributions 3 in $d+1$-dimensional configuration space $\mathbb{R}_{d+1}$, which are square integrable with respect to the scalar product (2.3).

Example 1. For the case of the free scalar field,

$$
S(x-y)=\frac{1}{(2 \pi)^{d+1}} \int d \bar{k} d k_{d+1} \frac{e^{i \bar{k} \cdot(\bar{x}-\bar{y})+i k_{d+1}\left(x_{d+1}-y_{d+1}\right)}}{k_{d+1}^{2}+\bar{k}^{2}+m^{2}}
$$

This last expression shows that the poles in the $k_{d+1}$ complex plane of $S(p)$, the propagator in momentum space, are located over the imaginary axis at $\pm i \omega_{m}(\bar{k})\left(\omega_{m}(\bar{k})=\sqrt{k^{2}+m^{2}}\right)$ in the $k_{d+1}$ complex plane. The quantity $\omega_{m}(\bar{k})$ correspond to the energy eigenvalues for this one particle system as will be shown below.

\footnotetext{
${ }^{3}$ This allows to consider point-like external sources.
} 
Let us consider the case when $f(x)=\sum_{j} q_{j} \delta(x-r(j)), q_{i} \in \mathbb{C}$ where $r(j)$ are the fixed positions of the "charge distribution"[11]. Using (2.3) leads to,

$$
\begin{aligned}
(\theta f, f) & =\int d^{d+1} x d^{d+1} y \sum_{i, j} q_{i}^{*} q_{j} \delta(\theta x-r(i)) S(x-y) \delta(y-r(j)) \\
& =\sum_{i, j} q_{i}^{*} q_{j} S(\theta r(i)-r(j)) \\
& =\int \frac{d \bar{k}}{(2 \pi)^{d}} \sum_{i, j} a_{i}^{*} a_{j} \int \frac{d k_{d+1}}{2 \pi} \frac{e^{-i k_{d+1}\left(r_{d+1}(i)+r_{d+1}(j)\right)}}{k_{d+1}^{2}+\bar{k}^{2}+m^{2}}
\end{aligned}
$$

where the $a_{i}$ are defined as follows,

$$
a_{i}=q_{i} e^{i \bar{k} \cdot \bar{r}(i)}
$$

the integral in the last equality in (2.4) can be done using the residue theorem, noting that $r_{d+1}(i)+r_{d+1}(j)>0$, a convenient contour is one that runs along the real axis and closes through a semicircle at infinity below the real axis, this leads to,

$$
(\theta f, f)=\int \frac{d \bar{k}}{(2 \pi)^{d}} \sum_{i, j} \bar{a}_{i} a_{j} \frac{e^{-\omega_{m}(\bar{k})\left(r_{d+1}(i)+r_{d+1}(j)\right)}}{2 \omega_{m}(\bar{k})}
$$

defining,

$$
b_{i}^{m}=a_{i} \frac{e^{-\omega(\bar{k}) r_{d+1}(i)}}{\sqrt{2 \omega(\bar{k})}}
$$

leads to,

$$
(\theta f, f)=\int \frac{d \bar{k}}{(2 \pi)^{d}} \sum_{i, j} \bar{b}_{i}^{m} b_{j}^{m}=\int \frac{d \bar{k}}{(2 \pi)^{d}} \sum_{i, j} \bar{b}_{i}^{m} T_{i j} b_{j}^{m}
$$

where the matrix $T_{i j}$ is a matrix with all entries equal to 1 . This matrix is positive semidefinite with eigenvalues given by,

$$
\operatorname{Eig}(T)=(N, 0, \cdots, 0)
$$

where $N$ is the dimension of the matrix.

Example 2. For the case of the higher derivative scalar field, $S(p)$ is given by,

$$
S_{h d}(p)=\frac{M^{2}-m^{2}}{\left(p^{2}+m^{2}\right)\left(p^{2}+M^{2}\right)}=\frac{1}{p^{2}+m^{2}}-\frac{1}{p^{2}+M^{2}}
$$

This last expression shows that the poles of propagator in momentum space $S_{h d}(p)$ in the complex $p_{d+1}$ plane, are located over the imaginary axis at $\pm i \omega_{m}(\bar{p})$ and at $\pm i \omega_{M}(\bar{p})$ $\left(\omega_{M}(\bar{p})=\sqrt{\bar{p}^{2}+M^{2}}\right)$. The quantities $\omega_{m}(\bar{p})$ and $\omega_{M}(\bar{p})$ correspond to the energy eigenvalues for this system as will be shown below. It is noted that in the limit $M \rightarrow \infty$, the previous example is obtained. 
Naively repeating the calculation as in the previous example, for $f(x)=\sum_{j} q_{j} \delta(x-r(j))$, one obtains,

$$
\begin{aligned}
(\theta f, f)_{h d} & =\int \frac{d \bar{k}}{(2 \pi)^{d}} \sum_{i, j} \bar{a}_{i} a_{j}\left(\frac{e^{-\omega_{m}(\bar{k})\left(r_{d+1}(i)+r_{d+1}(j)\right)}}{2 \omega_{m}(\bar{k})}-\frac{e^{-\omega_{M}(\bar{k})\left(r_{d+1}(i)+r_{d+1}(j)\right)}}{2 \omega_{M}(\bar{k})}\right) \\
& =\int \frac{d \bar{k}}{(2 \pi)^{d}}\left(\sum_{i, j} \bar{b}_{i}^{m} T_{i j} b_{j}^{m}-\sum_{i, j} \bar{b}_{i}^{M} T_{i j} b_{j}^{M}\right)
\end{aligned}
$$

, this expression is not positive definite. In this respect it is worth noting that the poles in the complex $p_{d+1}$-plane of $S_{h d}(p)$, indicate that two different particles are described by this correlator. If sources are included only for the particle with mass $m$ then reflection positive holds, however the other pole corresponds to a scalar free particle of mass $M$ that does not satisfy reflection positivity, it is a "ghost" with negative norm.

Example 3. Free scalar field on non-integer dimensional space[7]. In this case,

$$
S_{n i d}(p)=\frac{\left(p^{2}+M^{2}\right)^{2 \alpha}}{p^{2}+m^{2}}, \alpha \in \mathbb{R}, \alpha \geq 0, m^{2}=M^{2} \frac{\alpha n}{1-2 \alpha}
$$

where $M$ is a infrared regulator, and $n \geq 2$ is a positive integer. This expression shows that $S_{n i d}(p)$ has poles in the complex $p_{d+1}$ plane over the imaginary axis at $\pm i \omega_{m}(\bar{p})\left(\omega_{m}(\bar{p})=\right.$ $\left.\sqrt{\bar{p}^{2}+m^{2}}\right)$. It also has two branch cuts starting at $p_{d+1}= \pm i \omega_{M}(\bar{p})$ and ending at $\pm i \infty$, which correspond to a branch cut in the $p^{2}$ complex plane on the negative real axis for 4 $p^{2}<-M^{2}$. This is similar to what happens in a interacting local theory when considering corrections to the two-point function. For example in a $\varphi^{3}$ theory the one-loop correction involving particles of mass $\mu$ has a branch cut over the negative real axis for $p^{2}<-4 \mu^{2}$. This is a consequence of the optical theorem because $-4 \mu^{2}$ is the $p^{2}$ value required to produce a pair of those particles. In section 4 this analogy will be used to generate nonlocal reflection positive Euclidean field theories.

Under the same assumptions as in the previous cases it holds that,

$$
\begin{aligned}
(\theta f, f)_{n i d} & =\int d^{d+1} x d^{d+1} y \sum_{i, j} q_{i} q_{j} \delta(\theta x-r(i)) S_{n i d}(x-y) \delta(y-r(j)) \\
& =\int \frac{d \bar{p}}{(2 \pi)^{d+1}} \sum_{i, j} \bar{a}_{i} a_{j} \int d p_{d+1} \frac{e^{-i p_{d+1}\left(r_{d+1}(i)+r_{d+1}(j)\right)}\left(p_{d+1}^{2}+\bar{p}^{2}+M^{2}\right)^{2 \alpha}}{p_{d+1}^{2}+\bar{p}^{2}+m^{2}} \\
& =\int \frac{d \bar{p}}{(2 \pi)^{d+1}} \sum_{i, j} \bar{a}_{i} a_{j} I(r)
\end{aligned}
$$

where $r=r_{d+1}(i)+r_{d+1}(j)>0$ and,

$$
a_{i}=q_{i} e^{i \bar{p} \cdot \bar{r}(i)}
$$

as before. The integral $I(r)$ over $p_{d+1}$ can be done by choosing a contour in the complex $p_{d+1}$ plane, that runs bellow the real axis and continues through a quarter of a circle at infinity on the lower half plane up to the negative imaginary axis, goes back and forth along this axis up to $-i \omega_{M}(\bar{p})$ and returns to the real axis through the other quarter of a circle at infinity on the lower half plane. The contribution of both quarters of circle

\footnotetext{
${ }^{4}$ It is worth noting that here $p$ denotes the Euclidean momentum.
} 
vanishes( because $r=r_{d+1}(i)+r_{d+1}(j)>0$ !). Circulating the contour in the clockwise sense, the contribution of the back and forth path along the negative imaginary axis gives,

$$
I_{\text {cut }}(r)=\int_{\omega_{M}(\bar{p})}^{\infty} d \rho(-2 \sin (2 \pi \alpha)) e^{-\rho r} \frac{\left(\rho^{2}-\omega_{M}(\bar{p})^{2}\right)^{2 \alpha}}{\left(\rho^{2}-\omega_{m}(\bar{p})^{2}\right)}
$$

which shows that for $0<\alpha<1 / 2$ the integrand in this expression is negative for any value of $\rho$ within the integration limits. The residue of the pole at $p_{d+1}=-i \omega_{m}(\bar{p})$ is,

$$
\begin{aligned}
& \operatorname{Res}\left[\frac{\left(p_{d+1}^{2}+\omega_{M}(\bar{p})^{2}\right)^{2 \alpha}}{\left(p_{d+1}^{2}+\omega_{m}(\bar{p})^{2}\right)} e^{-i p_{d+1} r}\right]_{p_{d+1}=-i \omega_{m}(\bar{p})}= \\
& =i \frac{\left(M^{2}-m^{2}\right)^{2 \alpha}}{2 \omega_{m}(\bar{p})} e^{-\omega_{m}(\bar{p}) r}
\end{aligned}
$$

leading to,

$$
\begin{aligned}
I(r) & =-2 \pi i \operatorname{Res}\left[\frac{\left(p_{d+1}^{2}+\omega_{M}(\bar{p})^{2}\right)^{2 \alpha}}{\left(p_{d+1}^{2}+\omega_{m}(\bar{p})^{2}\right)} e^{-i p_{d+1} r}\right]_{p_{d+1}=-i \omega_{m}(\bar{p})}-I_{c u t} \\
& =2 \pi \frac{\left(M^{2}-m^{2}\right)^{2 \alpha}}{2 \omega_{m}(\bar{p})} e^{-\omega_{m}(\bar{p}) r}-I_{c u t}
\end{aligned}
$$

which shows that $I>0$ for $M^{2}-m^{2}>0$. Defining,

$$
b_{i}=a_{i}\left(\frac{\left(M^{2}-m^{2}\right)^{2 \alpha}}{2 \sqrt{\bar{p}^{2}+m^{2}}}\right)^{\frac{1}{2}} e^{-\omega_{m}(\bar{p}) r_{d+1}(i)}, b_{i}(\rho)=a_{i} e^{-\rho r_{d+1}(i)} \sqrt{\frac{\left(\rho^{2}-\omega_{M}(\bar{p})^{2}\right)^{2 \alpha}}{\left(\rho^{2}-\omega_{m}(\bar{p})^{2}\right)}}
$$

leads to,

$$
(\theta f, f)_{n i d}=\int \frac{d \bar{p}}{(2 \pi)^{d}}\left(\sum_{i, j} \bar{b}_{i} T_{i j} b_{j}+\int_{\omega_{M}(\bar{p})}^{\infty} d \rho \sum_{i, j} \bar{b}_{i}(\rho) T_{i j} b_{j}(\rho)\right)
$$

using the same argument as the one employed for the case of the free scalar field, the two terms in the r.h.s. of the last equation are positive definite for $M^{2}-m^{2}>0$. Using (2.5) this last condition implies that $\alpha<\frac{1}{n+2}$, and therefore for these $\alpha$ values reflection positivity holds for the free scalar field in a NIDS.

Below an alternative proof of reflection positivity for the scalar field on a non-integer dimensional space is presented. Using Newtons binomial series the integral $I$ appearing in (2.6) can be written as follows,

$$
I(r)=\int_{-\infty}^{\infty} d p_{d+1} e^{-i p_{d+1} r} \sum_{j=0}^{\infty}\left(\begin{array}{c}
2 \alpha \\
j
\end{array}\right)\left(p_{d+1}^{2}+\omega_{m}\right)^{2 \alpha-j-1}\left(\omega_{M}(\bar{p})^{2}-\omega_{m}(\bar{p})^{2}\right)^{j}
$$

where

$$
\left(\begin{array}{c}
2 \alpha \\
j
\end{array}\right)=\frac{2 \alpha(2 \alpha-1) \cdots((2 \alpha-j+1))}{j !}
$$

and $r=r_{d+1}(i)+r_{d+1}>0$. The binomial series inside the integral is uniformly convergent for $p_{d+1}^{2}>M^{2}-2 m^{2}$, therefore by analytic continuation it coincides and converges uniformly to $\left(p_{d+1}^{2}+\omega_{M}^{2}\right)^{2 \alpha}$ in the whole analyticity domain of this function, which includes the whole real axis. Thus integration and summation can be interchanged. Using that,

$$
\int_{-\infty}^{\infty} d p_{d+1} e^{-i p_{d+1} r}\left(p_{d+1}^{2}+\omega_{m}\right)^{2 \alpha-j-1}=\frac{2^{2 \alpha-j+\frac{1}{2}} \sqrt{\pi}}{\Gamma(j+1-2 \alpha)} K_{2 \alpha-j-\frac{1}{2}}\left(r \omega_{m}\right)\left(\frac{r}{\omega_{m}}\right)^{j-2 \alpha+\frac{1}{2}}
$$


leads to,

$$
I(r)=\sum_{j=0}^{\infty}\left(\begin{array}{c}
2 \alpha \\
j
\end{array}\right)\left(M^{2}-m^{2}\right)^{j} \frac{2^{2 \alpha-j+1 / 2} \sqrt{\pi}}{\Gamma(j+1-2 \alpha)} K_{2 \alpha-j-1 / 2}\left(r \omega_{m}\right)\left(\frac{r}{\omega_{m}}\right)^{j-2 \alpha+1 / 2}
$$

where $K_{\nu}(r)$ denotes the modified Bessel function such that $\lim _{r \rightarrow \infty} K_{\nu}(r)=0$. For $M^{2}-$ $m^{2}>0$ each term in this summation is positive thus implying that $I(r)>0$ in this case, using the same argument appearing after (2.9) in the previous proof, shows that reflection positivity holds for $M^{2}-m^{2}>0$, i.e. for $\alpha<\frac{1}{n+2}$.

For $\alpha<0$, which implies $d<n$, RP does not hold. This result may be shown using the same methods as above. However, it is important to note that for $\alpha<0$, the Fourier transform appearing in (2.5) presents additional poles. In order to apply the same calculation as in the $\alpha>0$ case, it is convenient to rewrite the Fourier transform as follows,

$$
S_{n i d}(p)=\frac{\left(p^{2}+M^{2}\right)^{1+2 \alpha}}{\left(p^{2}+m^{2}\right)\left(p^{2}+M^{2}\right)}, \alpha \in \mathbb{R}, \alpha<0, m^{2}=M^{2} \frac{\alpha n}{1-2 \alpha}
$$

where now $1+2 \alpha$, the exponent in the numerator, is positive. Recalling the previous example, this last expression shows that negative norm additional poles in the $p_{d+1}$-plane appear at $\pm i \omega_{M}(\bar{p})\left(\omega_{M}(\bar{p})=\sqrt{\bar{p}^{2}+M^{2}}\right)$. These poles spoil RP for $\alpha<0$.

\section{SCALAR PRODUCT AND ENERGY}

The pre-Hilbert5 1 -particle space $\mathcal{H}$, for a theory defined in Minkowski space is the one of tempered distributions over $\mathbb{R}_{d+1}$ with support on a fixed $x_{d+1}=x_{d+1}^{0}$ slice, i.e. of the form,

$$
f(x)=f(\bar{x}) \delta\left(x_{d+1}-x_{d+1}^{0}\right)
$$

equipped with the scalar product,

$$
<g, f>=(\theta g, f),
$$

this scalar product is positive semi-definite by virtue of RP. Taking $x_{d+1}^{0}=0$, gives,

$$
<g, f>=\int d \bar{x} d \bar{y} g^{*}(\bar{x}) S((0, \bar{x})-(0, \bar{y})) f(\bar{y})
$$

which in terms of the d-dimensional Fourier transforms of $g^{*}(\bar{x}), f(\bar{y})$ and $S((0, \bar{x})-(0, \bar{y}))$ is given by,

$$
<g, f>=\int \frac{d \bar{k}}{(2 \pi)^{d}} g^{*}(\bar{k}) S(\bar{k}) f(\bar{k})
$$

The energy eigenvalues can be obtained using the scalar product by means of,

$$
\begin{aligned}
<g, H f> & =-\left.\frac{d}{d \tau}\left(g, \theta T_{\tau} f\right)\right|_{\tau \rightarrow 0^{+}} \\
& =\int d \bar{x} d \bar{y} g^{*}(\bar{x})\left[-\frac{d}{d \tau} S((0, \bar{x})-(\tau, \bar{y}))\right]_{\tau \rightarrow 0^{+}} f(\bar{y})
\end{aligned}
$$

\footnotetext{
${ }^{5}$ This is not a Hilbert because the inner product defined above is positive semi-definite. To get a Hilbert space a quotient by the zero norm states should be done.
} 
where $T_{\tau}$ is the traslation by $\tau$ in the $d+1$ direction 6 , i.e.,

$$
T_{\tau} f\left(x_{d+1}, \bar{x}\right)=f\left(x_{d+1}-\tau, \bar{x}\right)
$$

which in terms of Fourier transforms is given by,

$$
<g, H f>=\left.\int \frac{d \bar{k}}{(2 \pi)^{d}} g^{*}(\bar{k}) D_{\tau} S(\bar{k}, \tau)\right|_{\tau \rightarrow 0^{+}} f(\bar{k})
$$

where $D_{\tau} S(\bar{k}, \tau)$ is defined by,

$$
-\frac{d}{d \tau} S((0, \bar{x})-(\tau, \bar{y}))=\int \frac{d \bar{k}}{(2 \pi)^{d}} D_{\tau} S(\bar{k}, \tau) e^{-i \bar{k} \cdot(\bar{x}-\bar{y})}
$$

The energy eigenvalues can be obtained using (3.2) and (3.4) for momentum eigenstates $f_{\bar{K}}(\bar{k})=\delta(\bar{k}-\bar{K})$ as follows,

$$
E(\bar{K})=\frac{<g, H f_{\bar{K}}>}{<g, f_{\bar{K}}>}
$$

Example 4. The free scalar field. Taking $x_{d+1}^{0}=0$ and noting that,

$$
\begin{aligned}
S((0, \bar{x})-(\tau, \bar{y})) & =\frac{1}{(2 \pi)^{d+1}} \int d \bar{k} d k_{d+1} \frac{e^{-i \bar{k} \cdot(\bar{x}-\bar{y})+i k_{d+1} \tau}}{k_{d+1}^{2}+\bar{k}^{2}+m^{2}} \\
& =\int \frac{d \bar{k}}{(2 \pi)^{d}} \frac{e^{-i \bar{k} \cdot(\bar{x}-\bar{y})} e^{-\omega_{m}(\bar{k}) \tau}}{2 \omega_{m}(\bar{k})}, \omega_{m}(\bar{k})=\sqrt{\bar{k}^{2}+m^{2}}
\end{aligned}
$$

leads in this case to the positive definite scalar product,

$$
<g, f>=\int \frac{d \bar{k}}{(2 \pi)^{d}} \frac{g(\bar{k})^{*} f(\bar{k})}{2 \omega_{m}(\bar{k})}
$$

The one-particle energy eigenvalues can be computed using (3.6), in order to do so it is noted that,

$$
\begin{aligned}
\left(g, \theta T_{\tau} f\right) & =\int d \bar{x} d^{d+1} y g^{*}(\bar{x}) S((0, \bar{x})-y) f(\bar{y}) \delta\left(y_{d+1}-\tau\right) \\
& =\int d \bar{x} d \bar{y} g^{*}(\bar{x}) S((0, \bar{x})-(\tau, y) f(\bar{y}) \\
& =\int \frac{d \bar{k}}{(2 \pi)^{d}} \frac{g(\bar{k})^{*} f(\bar{k})}{2 \omega_{m}(\bar{k})} e^{-\omega_{m}(\bar{k}) \tau}
\end{aligned}
$$

for the case of a plane wave $f(\bar{k})=\delta(\bar{k}-\bar{K})$, this leads to,

$$
\frac{<g, H f>}{<g, f>}=\frac{\int \frac{d \bar{k}}{(2 \pi)^{d}} g(\bar{k})^{*} f(\bar{k})}{\int \frac{d \bar{k}}{(2 \pi)^{d}} \frac{g(\bar{k})^{*} f(\bar{k})}{2 \omega_{m}(\bar{k})}}=\omega_{m}(\bar{K})
$$

Example 5. Higher derivative scalar field. Separating the propagator in the two parts,

$$
S_{h d}(p)=S_{h d}^{(m)}(p)+S_{h d}^{(M)}(p), S_{h d}^{(m)}(p)=\frac{1}{p^{2}+m^{2}}, S_{h d}^{(M)}(p)=-\frac{1}{p^{2}+M^{2}}
$$

\footnotetext{
${ }^{6}$ Althought this construction is aimed at defining the theory in Minkowki space, for the sake of simplicity, the Euclidean notation will still be employed in what follows.
} 
then the calculation for each pole is the same as in the previous case, leading to the following scalar products and energies,

$$
\begin{aligned}
& <g, f>_{m}=\int \frac{d \bar{k}}{(2 \pi)^{d}} \frac{g(\bar{k})^{*} f(\bar{k})}{2 \omega_{m}(\bar{k})}, E_{m}=\omega_{m}(\bar{k}) \\
& <g, f>_{M}=-\int \frac{d \bar{k}}{(2 \pi)^{d}} \frac{g(\bar{k})^{*} f(\bar{k})}{2 \omega_{m}(\bar{k})}, E_{M}=\omega_{M}(\bar{k})
\end{aligned}
$$

which shows that $S_{h d}^{(M)}(p)$ leads to a negative scalar product in accordance with the fact that for this part reflection positivity does not hold.

It is important to note that if this separation of the contribution for each pole is not done then the result for the energy eigenvalues is wrong. Indeed using the whole propagator one gets,

$$
\left(g, \theta T_{\tau} f\right)=\int \frac{d \bar{k}}{(2 \pi)^{d}} g(\bar{k})^{*} f(\bar{k})\left(\frac{e^{-\omega_{m}(\bar{k}) \tau}}{2 \omega_{m}(\bar{k})}-\frac{e^{-\omega_{M}(\bar{k}) \tau}}{2 \omega_{M}(\bar{k})}\right)
$$

which implies,

$$
-\left.\frac{d}{d \tau}\left(g, \theta T_{\tau} f\right)\right|_{\tau \rightarrow 0^{+}}=0 \Rightarrow H=0
$$

Example 6. Free scalar field on non-integer dimensional space. Using the results in example 3 leads to,

$$
S_{\text {nid }}((0, \bar{x})-(\tau, \bar{y}))=\int \frac{d \bar{p}}{(2 \pi)^{d+1}} I(\tau)
$$

The expression for $I(\tau)$ given in (2.9) will be employed in this calculation, i.e.,

$$
\begin{aligned}
I(\tau)= & \pi \frac{\left(M^{2}-m^{2}\right)^{2 \alpha}}{\omega_{m}(\bar{p})} e^{-\omega_{m}(\bar{p})|\tau|}+2 \sin (2 \pi \alpha) \\
& \times \int_{\omega_{M}(\bar{p})}^{\infty} d \rho e^{-\rho \tau} \frac{\left(\rho^{2}-\omega_{M}(\bar{p})^{2}\right)^{2 \alpha}}{\left(\rho^{2}-\omega_{m}(\bar{p})^{2}\right)}
\end{aligned}
$$

The integral appearing in the r.h.s. of the last equation can be explicitely computed by expanding the integrand as follows,

$$
\begin{aligned}
I(\tau)= & \pi \frac{\left(M^{2}-m^{2}\right)^{2 \alpha}}{\omega_{m}(\bar{p})} e^{-\omega_{m}(\bar{p})|\tau|}+2 \sin (2 \pi \alpha) \\
& \times \int_{\omega_{M}}^{\infty} d \rho \sum_{k=0}^{\infty} \frac{(-\rho \tau)^{k}}{k !} \sum_{j=0}^{\infty}\left(\begin{array}{c}
2 \alpha \\
j
\end{array}\right) \frac{\left(-\omega_{M}^{2}\right)^{j} \rho^{2(2 \alpha-j)}}{\rho^{2}-\omega_{m}^{2}}
\end{aligned}
$$

the two infinite series appearing inside the integral in the r.h.s. of the last equation are uniformly convergent, the first one converges to $e^{-\rho \tau}$ and the other is uniformly convergent for $\rho^{2}>\omega_{M}^{2}$ which is the integration interval. Therefore the order of integration and summation can be interchanged. The resulting integrals can be computed leading to,

$$
I(\tau)=\pi \frac{\left(M^{2}-m^{2}\right)^{2 \alpha}}{\omega_{m}(\bar{p})} e^{-\omega_{m}(\bar{p})|\tau|}+\omega_{m}(\bar{p})^{4 \alpha-1} \sum_{k=0}^{\infty} \frac{\left(-\tau \omega_{m}(\bar{p})\right)^{k}}{k !} I(k, \bar{p})
$$


where the adimensional quantity $I(k, \bar{p})$ is given by,

$$
\begin{aligned}
I(k, \bar{p})= & \sin (2 \pi \alpha) \sum_{j=0}^{\infty}\left(\begin{array}{c}
2 \alpha \\
j
\end{array}\right)\left(-\frac{\omega_{M}(\bar{p})}{\omega_{m}(\bar{p})}\right)^{2 j} \\
& \times \operatorname{Beta}\left(\frac{\omega_{m}(\bar{p})^{2}}{\omega_{M}(\bar{p})^{2}},-\frac{(4 \alpha+k-2 j-1)}{2}, 0\right)
\end{aligned}
$$

where Beta denotes Euler's beta function. Using these results in eqs. (3.2), (3.4) and replacing in (3.6) leads to,

$$
E(\bar{K})=\omega_{m}(\bar{K})\left(\frac{\pi\left(M^{2}-m^{2}\right)^{2 \alpha} \omega_{m}(\bar{K})^{-4 \alpha}+I(1, \bar{K})}{\pi\left(M^{2}-m^{2}\right)^{2 \alpha} \omega_{m}(\bar{K})^{-4 \alpha}+I(0, \bar{K})}\right)
$$

Expanding the quantity multiplying $\omega_{m}(\bar{K})$ in the r.h.s. of this last expression in powers of $\alpha$ leads to,

$$
E(\bar{K})=\omega_{m}(\bar{K})\left[1-2 \alpha \operatorname{Beta}\left(\frac{\bar{K}^{2}}{\bar{K}^{2}+M^{2}}, \frac{1}{2}, 0\right)+\mathcal{O}\left(\alpha^{2}\right)\right]
$$

to this order the rest mass is $m$, i.e. $E(0)=m$. However at higher orders there are non-vanishing corrections to this last equation.

\section{NON-LOCAL REFLECTION POSITIVE THEORIES}

Motivated by the similarity mentioned in example 3 in section 2 the following question is addressed. Under what conditions the corrected Euclidean propagator coming from a local interacting field theory leads to a scalar product, defined as in (3.3), satisfying the requirement of reflection positivity? It is stressed that the interest is not in whether the underlying local field theory satisfies reflection positivity or not, but on whether a nonlocal field theory defined by a non-local action obtained from the inverse of the corrected propagator satisfies reflection positivity or not. In order to answer the question above, the corrected Euclidean propagator is considered in greater detail. It is given by,

$$
S_{c}(p)=\frac{1}{p^{2}+m^{2}-\Sigma\left(p^{2}\right)}
$$

where in the perturbative case $\Sigma\left(p^{2}\right)$ has an expansion in powers of a coupling constant $g$ of the form,

$$
\Sigma\left(p^{2}\right)=g \Sigma_{1}\left(p^{2}\right)+g^{2} \Sigma_{2}\left(p^{2}\right)+\cdots .
$$

The propagator in coordinate space is given by,

$$
S_{c}(x-y)=\frac{1}{(2 \pi)^{d+1}} \int d \bar{p} d p_{d+1} \frac{e^{i \bar{p} \cdot(\bar{x}-\bar{y})+i p_{d+1}\left(x_{4}-y_{4}\right)}}{p_{d+1}^{2}+\bar{p}^{2}+m^{2}-\Sigma\left(p^{2}\right)}
$$

It is useful to consider the expansion of $\Sigma\left(p^{2}\right)$ around $p^{2}=-m^{2}$, which leads to the following identity,

$$
p^{2}+m^{2}-\Sigma\left(p^{2}\right)=p^{2}+m^{2}-\Sigma\left(-m^{2}\right)-\Sigma^{\prime}\left(-m^{2}\right)\left(p^{2}+m^{2}\right)-\frac{\Sigma^{\prime \prime}\left(-m^{2}\right)}{2 !}\left(p^{2}+m^{2}\right)^{2}-\cdots
$$

the equation,

$$
p^{2}+m^{2}-\Sigma\left(p^{2}\right)=0
$$

enables to compute the poles of $S_{c}(p)$. This equation together with (4.1) shows that the location of these poles is such that $p^{2}+m^{2} \simeq \mathcal{O}(g)$. Thus up to $\mathcal{O}\left(g^{2}\right)$ the poles in the 
$p_{d+1}$ complex plane can be obtained solving the following equation, whose solutions are also shown below,

$$
p^{2}+m^{2}-\Sigma\left(-m^{2}\right)=0 \Rightarrow p_{d+1}= \pm i\left(\omega(\bar{p})-\frac{\Sigma\left(-m^{2}\right)}{2 \omega(\bar{p})}\right) .
$$

Evaluating the integral over $p_{d+1}$ using residues leads to,

$$
S_{c}(x-\theta y)=\frac{i}{(2 \pi)^{d}} \int d \bar{p} e^{-i \bar{p} \cdot(\bar{x}-\bar{y})} \frac{e^{-\left(\omega(\bar{p})-\frac{\Sigma\left(-m^{2}\right)}{2 \omega(\bar{p})}\right)\left(x_{4}-\theta y_{4}\right)}}{2 i\left(\omega(\bar{p})-\frac{\Sigma\left(-m^{2}\right)}{2 \omega(\bar{p})}\right)}, x_{4}>0, y_{4}<0
$$

using the same arguments as in the previous cases it is concluded that RP will hold whenever the quantity $\omega(\bar{p})-\frac{\Sigma\left(-m^{2}\right)}{2 \omega(\bar{p})}$ is real and greater than zero. If $\Sigma\left(-m^{2}\right)$ is real then this will be the case because in the perturbative regime $\Sigma\left(-m^{2}\right) \ll \omega(\bar{p})^{2}$. However if $\Sigma\left(-m^{2}\right)$ has a imaginary part then RP would not hold. As is well known, this can happen even if the underlying local field theory satisfies RP. Indeed in that case such a imaginary part will appear if the particle whose corrected propagator is considered can decay through a real process into other particles[12, i.e. if it is a unstable particle. This is a consequence of the optical theorem for the underlying local RP theory. In addition, there exists a reduction to the absurd argument leading to the same result. Suppose that RP would hold for the corrected propagator, that would imply that the time evolution for this corrected particle is unitary, however this is not true since the $S$-matrix is not unitary for a theory including unstable asymptotic particles[12]. Furthermore, this makes sense from the physical point of view, if the particle can decay then its energy contains an imaginary part and the time evolution restricted to this one unstable particle subspace is not unitary.

It is possible to go a step further in the analogy between the corrected propagator and the free theory in a NIDS. The two point function for the later case can be written as follows,

$$
\begin{aligned}
S_{n i d}(p) & =\frac{\left(p^{2}+M^{2}\right)^{2 \alpha}}{p^{2}+m^{2}}=\frac{1}{\left(p^{2}+m^{2}\right) e^{-2 \alpha \log \left(p^{2}+M^{2}\right)}} \\
& =\frac{1}{p^{2}+m^{2}-\Sigma^{(n i d)}\left(p^{2}\right)}
\end{aligned}
$$

where,

$$
\Sigma^{(n i d)}\left(p^{2}\right)=\alpha \Sigma_{1}^{(n i d)}\left(p^{2}\right)+\alpha^{2} \Sigma_{2}^{(n i d)}\left(p^{2}\right)+\cdots
$$

with,

$$
\Sigma_{1}^{(n i d)}\left(p^{2}\right)=-2\left(p^{2}+m^{2}\right) \log \left(p^{2}+M^{2}\right), \Sigma_{2}^{(n i d)}\left(p^{2}\right)=2\left(p^{2}+m^{2}\right) \log \left(p^{2}+M^{2}\right)^{2}
$$

comparing (4.1) with (4.2) shows that the departure $\alpha$ from the integer dimensional case plays the role of a coupling constant in this analogy.

\section{CONCLUding REMARKS}

In this work the requirement of RP has been studied in some particular cases. This study leads to the following conclusions,

- The pole structure of the corresponding two point functions encodes the states present in the theory. In particular, the case of the higher derivative scalar field is a clear illustration that in order to understand the spectrum it is convenient 
(when possible) to separate the Fourier transformed two point function in single pole terms.

- The scalar theory defined on a NIDS satisfies RP for $0<\alpha<\frac{1}{n+2}$, i.e. for $n<d$. This result is extremely interesting because it implies that a field theory on a NIDS produces a unitary evolution. Having a unitary evolution for a field theory on a NIDS space permits to address the question of whether physical space has integer dimension or not.

On the other hand for $\alpha<0$, i.e. for $d<n \mathrm{RP}$ does not hold. This result agrees partially with the one in [13], however in this respect it is important to realize that the NIDS employed in both works are not the same.

- The appearance of a branch cut for the two point function in momentum space for the NIDS has suggested an analogy with an interacting perturbative local field theory. In this analogy, the deformation parameter 7 in the NIDS plays the role the coupling constant in the interacting theory. The question of whether there exists interacting perturbative local field theories leading to the same two point function as the free theory on the NIDS remains open. It has been shown that the corrected propagator of a local unitary field theory leads to a reflection positive Euclidean non-local theory whenever the particle whose corrected propagator is considered can not decay through a real process into other particles.

\section{Acknowledgments.}

I would like to thank H. Casini for explaining to me the relation between RP and unitarity, and also for a useful critical reading of the manuscript. I also thank G. Torroba for encouraging discussions on the subject and to S. Grillo for his mathematical support. I appreciate discussions with S. Rychkov.

\section{APPENDIX.}

Spectral functions. The spectral function $\rho$ is related to the Fourier transform of the two-point function $S\left(p^{2}\right)$ (a recent review of the subject appears in [14]). It will be assumed that the singularities of the Fourier transform of the Euclidean two-point function are given by a pole at $p^{2}=-m^{2}$ and a cut starting at a certain real negative value $-\mu^{2}$ of $p^{2}$. Using a keyhole contour $\gamma$ consisting of a circle at infinity and a back and forth path going above and below the negative real axis starting at the first singularity to the right, $S\left(p^{2}\right)$ can be written as follows,

$$
S\left(p^{2}\right)=\frac{1}{2 \pi i} \int_{\gamma} d s \frac{S(s)}{s-p^{2}}
$$

assuming that $S\left(p^{2}\right)$ is such that the circle at infinity does not contribute to the integral in the last equation, then,

$$
S\left(p^{2}\right)=\frac{-1}{2 \pi i} \lim _{\epsilon \rightarrow 0^{+}} \int_{-s_{0}}^{-\infty} d s \frac{S(s+i \epsilon)-S(s-i \epsilon)}{s-p^{2}}
$$

\footnotetext{
${ }^{7}$ This quantity parametrizes the deviation from the integer dimensional case, as shown by equation (1.1).
} 
where $-s_{0}$ is a value of $s$ such that no singularities appear for $s>-s_{0}$. The quantity multiplying $\frac{1}{s-p^{2}}$ is the spectral function 8 , i.e.,

$$
\rho(s)=-\frac{1}{2 \pi i}\left(\lim _{\epsilon \rightarrow 0^{+}} S(s+i \epsilon)-S(s-i \epsilon)\right)
$$

Reflection positivity in terms of the spectral function is the requirement that,

$$
\rho(s) \geq 0, s \in \mathbb{R} .
$$

Bellow the case of the scalar field in the NIDS is considered. In this case the circle at infinity does not contribute. Using (5.2) or (5.1) leads to,

$$
\begin{aligned}
\rho(s)= & -\frac{1}{\pi} \lim _{\epsilon \rightarrow 0^{+}} \operatorname{Im}\left[S_{n i d}(s+i \epsilon)\right]=-\frac{1}{2 \pi i}\left(\lim _{\epsilon \rightarrow 0^{+}} S(s+i \epsilon)-S(s-i \epsilon)\right) \\
= & \frac{1}{\pi} \lim _{\epsilon \rightarrow 0^{+}} \frac{\epsilon\left(\left(s+M^{2}\right)^{2}+\epsilon^{2}\right)^{\alpha}}{\left(s+m^{2}\right)^{2}+\epsilon^{2}} \\
& \left(\left(s+m^{2}+i \epsilon\right) e^{i 2 \alpha \arctan \left(\frac{\epsilon}{s+M^{2}}\right)}-\left(s+m^{2}-i \epsilon\right) e^{-i 2 \alpha \arctan \left(\frac{\epsilon}{s+M^{2}}\right)}\right) \\
= & \frac{1}{\pi} \lim _{\epsilon \rightarrow 0^{+}} \frac{\epsilon\left(\left(s+M^{2}\right)^{2}+\epsilon^{2}\right)^{\alpha}}{\left(s+m^{2}\right)^{2}+\epsilon^{2}} \times \\
& \times\left(\epsilon \cos \left(2 \alpha \arctan \left(\frac{\epsilon}{s+M^{2}}\right)\right)-\left(s+m^{2}\right) \sin \left(2 \alpha \arctan \left(\frac{\epsilon}{s+M^{2}}\right)\right)\right.
\end{aligned}
$$

which satisfies RP for $M^{2}>m^{2}$, since in that case it is positive semi-definite for $s \in \mathbb{R}$, $\epsilon \ll 1$ and $\epsilon>0$.

\section{REFERENCES}

[1] Konrad Osterwalder and Robert Schrader. Axioms for euclidean green's functions. Communications in Mathematical Physics, 31(2):83-112, 1973.

[2] K. G. Wilson and John B. Kogut. The Renormalization group and the epsilon expansion. Phys. Rept., 12:75-200, 1974.

[3] CG Bollini and JJ Giambiagi. Dimensional renorinalization: The number of dimensions as a regularizing parameter. Il Nuovo Cimento B (1971-1996), 12(1):20-26, 1972.

[4] Gerard 't Hooft and M. J. G. Veltman. Regularization and Renormalization of Gauge Fields. Nucl. Phys., B44:189-213, 1972.

[5] A. Connes. Noncommutative Geometry. Elsevier Science, 1995.

[6] Erik Christensen, Cristina Ivan, and Michel L. Lapidus. Dirac operators and spectral triples for some fractal sets built on curves. Advances in Mathematics, 217(1):42 - 78, 2008.

[7] R. Trinchero. Scalar field on non-integer dimensional spaces. Int. J. Geom. Meth. Mod. Phys., 09:1250070, 2012.

[8] J. Glimm and A. Jaffe. Quantum Physics: A Functional Integral Point of View. Springer-Verlag, 1987.

[9] Francesco Guerra. Euclidean field theory. In Encyclopedia of Mathematical Physics, pages 256-265. Elsevier Limited, Oxford, 2006, 2006.

[10] C.C. Anderson. Defining Physics at Imaginary Time: Reflection Positivity for Certain Riemannian Manifolds. Harvard University, 2013.

\footnotetext{
${ }^{8}$ For the case in which the two-point function is such that $S(s)$ is real for $s$ in a open interval of the
} real line, then Schwarz reflection principle implies that,

$$
\rho(s)=-\frac{1}{2 \pi i}\left(\lim _{\epsilon \rightarrow 0^{+}} S(s+i \epsilon)-S(s-i \epsilon)\right)=-\frac{1}{\pi} \lim _{\epsilon \rightarrow 0} \operatorname{Im}[S(s+i \epsilon)]
$$


[11] A. Uhlmann. Some remarks on reflection positivity. Czechoslovak Journal of Physics B, 29(1):117-126, 1979.

[12] M. J. G. Veltman. Unitarity and causality in a renormalizable field theory with unstable particles. Physica, 29:186-207, 1963.

[13] M. Hogervorst, S. Rychkov, and B. C. van Rees. Unitarity violation at the Wilson-Fisher fixed point in $4-\epsilon$ dimensions. Phys. Rev. D, 93(12):125025, June 2016.

[14] Roman Zwicky. A brief Introduction to Dispersion Relations and Analyticity. In Quantum Field Theory at the Limits: from Strong Fields to Heavy Quarks (HQ 2016) Dubna, Russia, July 18-30, 2016, 2016. 